\title{
Lateral minimal approach to the terrible triad of the elbow: a treatment protocol in Beijing Jishuitan Hospital
}

\author{
Ye-Jun Zha, Dan Xiao, Ke-Han Hua, Wei-Tong Sun, Mao-Qi Gong, Ting Li, Chen Chen, Xie-Yuan Jiang \\ Department of Orthopedic Trauma, Beijing Jishuitan Hospital, Beijing, China \\ Contributions: (I) Conception and design: YJ Zha; (II) Administrative support: YJ Zha, XY Jiang; (III) Provision of study materials or patients: D Xiao, \\ MQ Gong; (IV) Collection and assembly of data: KH Hua, WT Sun; (V) Data analysis and interpretation: T Li, C Chen; (VI) Manuscript writing: \\ All authors; (VII) Final approval of manuscript: All authors. \\ Correspondence to: Xie-Yuan Jiang. Beijing Jishuitan Hospital, 31 Xinjiekou East Rd, Xicheng District, Beijing 100035, China. Email: jxy0845@sina.com.
}

Background: This study aimed to report the surgical techniques and results of treating coronoid process and radial head fracture combined with dislocation of the elbow (terrible triad of the elbow) using a single lateral incision, known as the extensor digitorum communis (EDC) split approach.

Methods: A retrospective analysis was performed of 109 patients with terrible triad of the elbow who had been treated by the authors from January 2013 to December 2019. The participants included 67 males and 42 females, with a mean age of 42.2 years (14-71 years). All participants were treated via a single lateral approach. The coronoid process was fixated with Kirschner wires combined with anterior capsule suture lasso fixation. For the radial head fracture, 58 cases were fixated by AO headless cannulated screw (AO HCS) and 51 cases by acumed radial head replacement. In repair of the lateral collateral ligament (LCL) complex and the common extensor tendon, 28 cases used ETHIBOND suture through bone holes at the humeral lateral epicondyle, and the other 81 cases used suture anchors. No medial collateral ligament was repaired. A total of 46 participants were fixated with a Stryker dynamic joint distractor (DJD) II hinged external fixator to protect the bone and soft tissue.

Results: All participants were followed up from 6 to 60 months (mean, 36.1 months). Their elbow range of flexion and extension averaged $123.4^{\circ} \pm 20.7^{\circ}$, forearm rotation $151.0^{\circ} \pm 25.6^{\circ}$, and Mayo elbow performance score (MEPS) 92.3 \pm 8.8 . There were 22 participants (19.5\%) with ulnar nerve symptoms, $16(14.7 \%)$ who had elbow stiffness, and 7 underwent secondary surgery, including 6 removals of internal fixation, 5 arthrolyses of the elbow, and 2 ulnar neurolyses.

Conclusions: Coronoid fractures, radial head fractures, and LCL injuries of the terrible triad of the elbow can be treated satisfactorily through a lateral minimal incision, combined with a hinged external fixation if necessary.

Keywords: Elbow; fractures; elbow dislocations; radial head; coronoid process

Submitted Mar 15, 2021. Accepted for publication Aug 01, 2021.

doi: $10.21037 /$ atm-21-2542

View this article at: https://dx.doi.org/10.21037/atm-21-2542

\section{Introduction}

The terrible triad of the elbow, which was first described by Hotchkiss in 1996, is usually characterized by elbow dislocation combined with fractures of the radial head and coronoid process of the ulna (1). Typically, the elbows display obvious posterolateral rotational instability and associated severe soft tissue injuries, especially the lateral collateral ligament (LCL) complex $(2,3)$. Therefore, the treatment of this complex injury has posed a great challenge for orthopedic surgeons and is often associated with devastating complications including elbow stiffness (10.3\%), failure of osteosynthesis $(6.7 \%)$ and ulnar neuropathy $(6.2 \%)$ etc. $(2,4-6)$. Recently, with more in-depth studies regarding elbow anatomy and biomechanics as well as the 


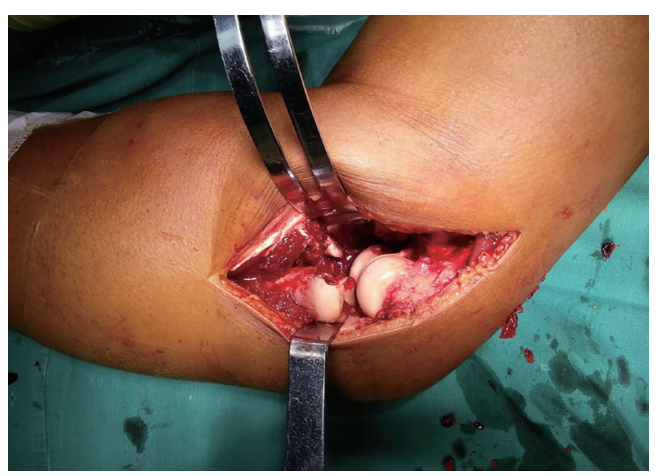

Figure 1 The single lateral incision.

advancement of surgical techniques, the prognosis of a such complex injury has been greatly improved. However, the sample sizes of previous clinical studies have been relatively small and the surgical procedures for treating the terrible triad have remained controversial, especially concerning the specific surgical approaches (7-10).

Regarding the surgical approaches, a posterior approach $(7,8)$ or a combined medial and lateral approach $(9,10)$ have been the preferred choices for most surgeons to restore elbow stability. However, these 2 approaches have been found to increase intraoperative soft tissue disruption to the elbow, and thus, they may increase the risk of developing postoperative elbow stiffness, heterotopic ossification, or ulnar nerve symptoms.

In this study, we proposed a treatment protocol for the terrible triad of the elbows using an extensor digitorum communis (EDC) split approach through a single lateral incision, which is generally $4 \mathrm{~cm}$ long with very few cases extending to $6 \mathrm{~cm}$. Here, we aimed to introduce this method in detail and further evaluate the clinical outcomes of patients with terrible triad treated via this surgical approach. We present the following article in accordance with the STROBE reporting checklist (available at https:// dx.doi.org/10.21037/atm-21-2542).

\section{Methods}

Patients in Beijing Jishuitan Hospital Department of Orthopedic Trauma with acute terrible triad of elbows who required surgical treatment due to severe instability or displaced fracture fragments after closed reduction between January 2013 and December 2019 were included in this study. Retrospective clinical research was conducted with the following inclusion criteria: (I) treated by single lateral incision and EDC split approach; (II) complete perioperative information and postoperative follow-up data; (III) minimum 1 year follow-up time. The exclusion criteria were as follows: (I) age $<14$ years old; (II) pathologic fractures; (III) unclosed epiphysis; and (IV) lost to follow-up.

A total of 136 patients with the terrible triad of the elbows underwent surgical treatment at our institution. After applying the inclusion and exclusion criteria, 109 patients were included in the study. For the patients who were excluded, there were 2 patients who were $<14$ years old, and there were 25 patients who were lost to follow-up. The mean age of all participants was $42.2 \pm 13.8$ [14-71] The study was approved by Beijing Jishuitan Hospital Institutional Review Board (No. 201708-04) and written informed consent was obtained from all patients. All procedures performed in this study involving human participants were in accordance with the Declaration of Helsinki (as revised in 2013).

All participants underwent closed reduction and immobilization with plaster in the emergency room to maximally restore joint congruence. All participants were treated via a single lateral incision, which was generally $4 \mathrm{~cm}$ (with very few cases extending to $6 \mathrm{~cm}$ ), and EDC split approach to expose the injured LCL complex, the origin of common extensor tendon, radial head fracture, coronal process fracture, and anterior joint capsule, respectively (Figure 1). Usually, a bare area was to be found on the posterior aspect of the lateral epicondyle of the distal humerus, due to the origin of the LCL complex having been torn apart. Through the "entrance" just distal to the bare area, the common extensor muscle was split open along the lateral epicondyle and the midline of the radial head. In this way, it was easier to avoid further disruption to collateral ligaments and surrounding muscles and preferable for the exposure and fixation of fractures of the radial head and coronoid process. However, the anterior aspect of the lateral epicondyle was usually intact and required additional stripping to enhance exposure.

We firstly dealt with fractures of the coronoid process. The anterior joint capsule attached to the coronoid fracture fragment was sutured using ETHIBOND\#2 (Ethicon Inc., Johnson and Johnson, New Brunswick, NJ, USA) and further reduced using the "lasso technique" without tightening the suture tails. Then, a pair of K-wires (diameter $1.5 \mathrm{~mm}$ ) were inserted from the dorsal side of the proximal ulna into the center of the base of the coronoid fragment. After adequate reduction of the coronoid process using the lasso, the K-wires were further inserted into the fragment 

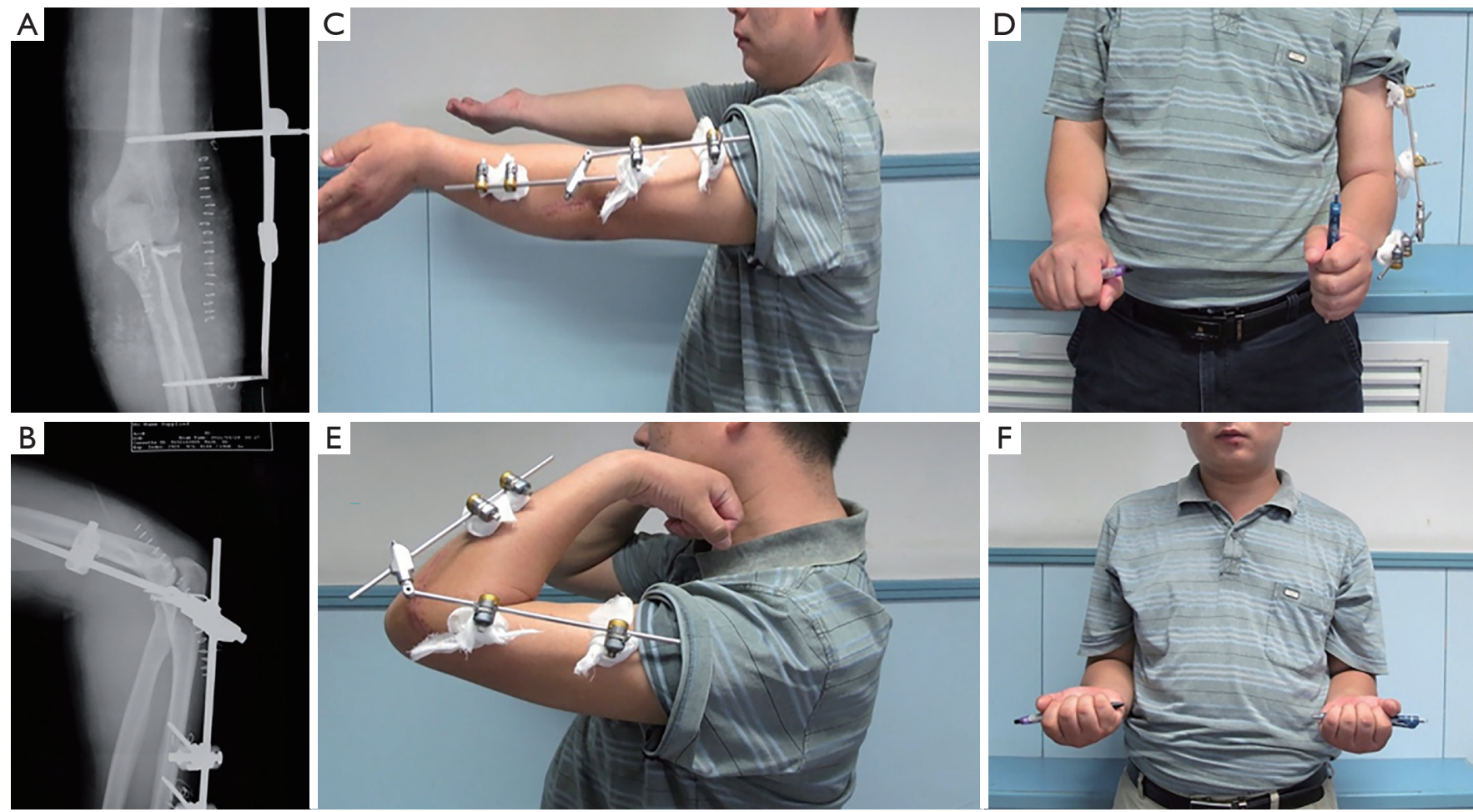

Figure 2 Elbow function and X-ray postoperatively. (A,B) The postoperative X-ray of a patient with Stryker DJD II hinged external fixator. (C-F) The ROM of the patient after 2 months. DJD, dynamic joint distractor; ROM, range of movement.

for stabilization. The suture tails were tensioned and tied on the subcutaneous posterior border of the ulna. Then, the tail of K-wires was bent, shortened, and buried beneath the skin. The sequential order of K-wire fixation and suture tail tightening is crucial for preventing the secondary fragmentation and loosening of the lasso suture. If the fragment was too small or comminuted to be fixed by K-wires, we just performed a "lasso technique" to stabilize the coronoid process.

Secondly, we dealt with the radial head fractures. A total of 58 participants were fixed by $2.4 \mathrm{~mm}$ headless cannulated screws (HCS), and the remaining 51 participants were treated by radial head replacement due to severe comminution.

Thirdly, we dealt with the soft tissue. The LCL complex and the origin of common extensor tendon were repaired using either suture anchor (81 participants) or transosseous braided suture through the lateral epicondyle using ETHIBOND\#2 (28 participants). In all participants, the medial collateral ligaments (MCL) were not surgically treated and left as they were.

Lastly, the elbow joint stability must be carefully examined after open reduction and internal fixation. Physical examination revealed mild "drop sign" on lateral radiographs when flexing the elbow joints in 46 participants, and they were further treated by Stryker dynamic joint distractor (DJD) II hinged external fixator (Stryker Corp., Kalamazoo, MI, USA) in order to achieve better joint stability and protect the fixation of bony structures and repair of soft tissue (Figure 2). This supplementary intervention was performed to enable patients to achieve full range of motion exercises in early stage rehabilitation. A total of 63 participants achieved adequate stability when flexing and extending the elbow joint during surgery, and they were protected by braces or plaster casts (for less than 1 week) as precautionary measures in order to maintain joint congruence (Figure 3). The humeroulnar joints of all our participants were not fixed by K-wires. Drainage was removed when there was less than $30 \mathrm{~mL}$ during the first 24-48 hours after surgery. Glucosamine was routinely administered $100 \mathrm{mg}$ TID for 6 weeks postoperatively. The $\mathrm{K}$-wires for the fixation of coronoid process fracture were usually removed after 2-3 months.

Regarding postoperative rehabilitation, for participants 

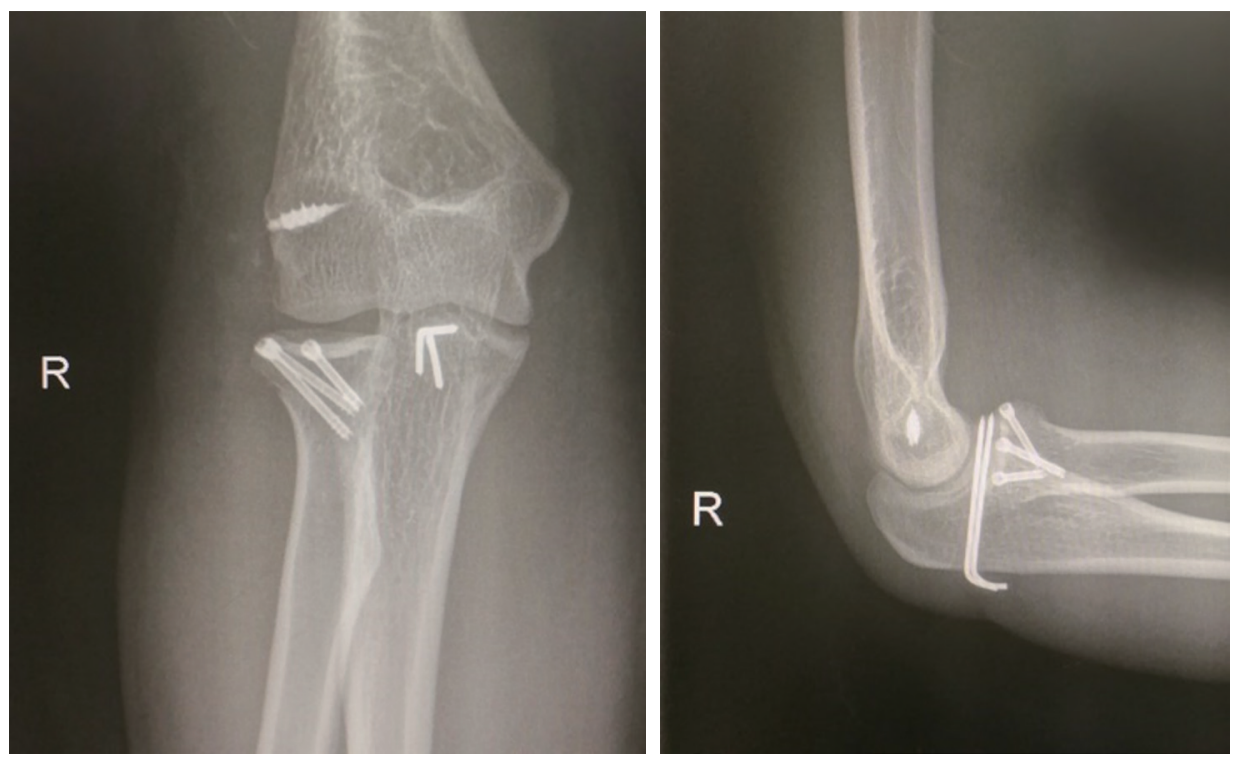

Figure 3 The postoperative X-ray without an external fixator.

treated by external fixators, full range of motion (ROM) active and gentle passive exercises were initiated on the second day after surgery. Participants without external fixators were protected by braces or casts for a week without active extension exercises. Moreover, elbow extension was limited to within 30 degrees during the first month of rehabilitation. Violent passive massage or stretching conducted by others were strictly forbidden.

Functional outcomes were collected and documented in our database after routine follow-ups in outpatient clinics. Clinical results of the latest follow-up were extracted from our database and evaluated using the parameters including chief complaints, ROM, Mayo Elbow Performance Score (MEPS) (11,12), visual analogue scale (VAS), complications, and secondary operations. The ROM and MEPS was measured by a doctor who wasn't the surgeon.

\section{Statistical analysis}

The software SPSS 24.0 (IBM Corp. Armonk, NY, USA) was used for statistical analysis of all follow-up data. For the quantitative variables, the descriptive statistics included means, medians, standard deviations, and ranges.

\section{Results}

A total of 109 patients with the terrible triad of the elbows were collected from our database. The average follow- up duration was $36.1 \pm 11.1$ months (6-60 months). The baseline characteristics were as follows (Table 1).

The average ROM of flexion and extension was $123.8^{\circ} \pm 20.5^{\circ}, \mathrm{ROM}$ of rotation was $151.1^{\circ} \pm 25.1^{\circ}$, MEPS was $92.4 \pm 8.8$ (68.8\% were excellent), and VAS was $0.9 \pm 1.5$. There were 22 participants $(19.5 \%)$ who experienced ulnar nerve symptoms, which is defined as local sensory abnormality or weakened muscle strength after surgery. There were 16 participants (14.7\%) with elbow stiffness, which is generally defined as an elbow ROM less than $100^{\circ}$ either in flexion-extension or pronation-supination. There were 7 participants who underwent secondary surgery, including 6 removals of internal fixation, 5 arthrolyses of the elbow, and 2 ulnar neurolyses. None of the participants experienced infection or bone nonunion (Table 2).

\section{Discussion}

The terrible triad of the elbow refers to complex fracture and dislocation of radial head fracture, coronoid fracture, and elbow dislocation, which often leads to complications such as elbow stiffness, failure of osteosynthesis, ulnar neuropathy and recurrent instability $(2,6)$.

The coronoid process and the radial head are the primary constraints against posterior translation of the forearm. After a fall on the outstretched hand with the elbow extended or slightly bent, the forearm translates posteriorly, which leads to transverse shearing fractures of the coronoid, 
Table 1 Participant baseline characteristics

\begin{tabular}{lc}
\hline Baseline characteristics & Results \\
\hline Age & $42.2 \pm 13.8$ \\
Gender & $67(61.5 \%)$ \\
Male & $42(38.5 \%)$ \\
Female & \\
Injury energy level & $21(19.3 \%)$ \\
High energy & $88(80.7 \%)$ \\
Low energy & \\
Treatment of radial head & $58(53.2 \%)$ \\
fixed by HCS & $51(46.8 \%)$ \\
radial head replacement & \\
Repair of LCL complex & $81(74.3 \%)$ \\
Using suture anchor & $28(25.7 \%)$ \\
Using ETHIBOND\#2 & \\
Postoperative external fixation & $46(42.2 \%)$ \\
Stryker DJD II hinged external fixator & $63(57.8 \%)$ \\
Braces or plaster casts &
\end{tabular}

HCS, headless cannulated screw; LCL, lateral collateral ligament; DJD, dynamic joint distractor.

and the "anterior rim" of the radial head hits against the capitulum causing a radial head fracture. As a result, the terrible triad injury occurs. With thorough understanding of elbow anatomy and biomechanics and the advancement of surgical techniques, orthopedists have gradually established systematic treatment protocols and rehabilitation strategies for the terrible triad, which has significantly improved its prognosis.

The goal of surgical management of the terrible triad is to achieve concentric reduction and stability of the elbow joint, which allows early mobilization to maximally restore elbow function (13). For less severe injuries, closed reduction and hinge external fixation can be used to achieve satisfactory results. Most instances of the terrible triad will lead to bony block and difficulty in maintaining the stability of the elbow, so treatment is usually via surgery. Common surgical approaches to the terrible triad include the posterior approach, combined medial and lateral approach, and anterior approach (7-10). Many surgeons may choose the first 2 approaches to reconstruct the anatomy. Lindenhovius et al. (14) followed 18 terrible triad patients through the posterior approach, achieving good functional outcomes
Table 2 The functional outcomes of participants

\begin{tabular}{lc}
\hline Variable & Results \\
\hline ROM of flexion and extension & $123.4^{\circ} \pm 20.7^{\circ}$ \\
ROM of rotation & $151.0^{\circ} \pm 25.6^{\circ}$ \\
MEPS & $92.3 \pm 8.8$ \\
MEPS rank & $75(68.8 \%)$ \\
Excellent (MEPS >90) & $34(31.2 \%)$ \\
Other & $0.9 \pm 1.5$ \\
VAS & \\
Complications & $22(19.5 \%)$ \\
Ulnar nerve symptoms & $16(14.7 \%)$ \\
Elbow stiffness & 6 \\
Secondary surgery & 5 \\
Removal of internal fixation & 2 \\
Arthrolysis of the elbow & $76.4 \%)$ \\
Ulnar neurolysis & \\
Total & \\
\hline ROM, range & \\
\hline
\end{tabular}

ROM, range of motion; MEPS, Mayo Elbow performance score; VAS, visual analogue scale.

with a flexion-extension arc of $119^{\circ}$ and a rotational range of $141^{\circ}$, and the average MEPS of these patients was 88 points, after a mean follow-up of 24 months. Zhang et al. (10) followed 21 terrible triad patients undergoing internal fixation through the combined medial and lateral approach for an average of 32 months, achieving good functional outcomes with a flexion-extension arc of $126.0^{\circ} \pm 4.8^{\circ}$ and a rotational range of $139.0^{\circ} \pm 4.1^{\circ}$. The average MEPS of these patients was 95.2 points. Although these 2 approaches can more satisfactorily restore the stability of the elbow joint, they significantly increase surgical trauma, which adds to the probability of joint stiffness, ectopic ossification, and ulnar nerve symptoms. The anterior approach exposes the coronoid process more optimally, but also damages the anterior joint capsule. Once stiffness occurs, it will be difficult to improve the extension of the elbow. Therefore, the anterior approach is not recommended. Pugh et al. (13) proposed a single lateral approach to the terrible triad, which achieved satisfactory outcomes. A year later, McKee et al. (15) suggested a standard surgical protocol for the terrible triad of the elbow, indicating that a single lateral approach was enough for most cases, and the combined 
medial approach was needed only when the coronoid process was difficult to expose by the lateral approach, preoperative ulnar nerve injuries existed, and MCL needed to be repaired.

At Beijing Jishuitan Hospital, we have treated the terrible triad of the elbow through a lateral minimal approach. Compared with the other abovementioned approaches, the single lateral minimal approach damages less soft tissue, maintaining the stability of the injured elbow, which may lead to better functional outcomes. Through the lateral minimal approach, the injured lateral ulnar collateral ligament (LUCL), radial head fracture, coronoid fracture, and anterior joint capsule were revealed from shallow to deep, and then the coronoid process, anterior joint capsule, radial head, LCL complex, and extensor tendon origin were repaired from deep to shallow. The lateral epicondyle was used as an anatomical landmark. A $4 \mathrm{~cm}$ incision was made along the line of the supracondylar crest-lateral epicondyleradial head. Soft tissue avulsion was usually found behind the midline of the lateral epicondyle, forming a bare area. Through the original rupture, the EDC was split along the lateral epicondyle of the humerus and the midline of the radial head. Dissection did extend distally to the radial neck, to avoid damage to the deep branch of the radial nerve below the radial tubercle. Proximal dissection of the brachioradialis and extensor carpi radialis longus and brevis anterior to the midline of the lateral epicondyle was performed, and the anterior joint capsule was retracted forward. Even if the radial head fracture was relatively intact with small fragments, the coronoid process could be well exposed and fixed through this approach. In cases treated with radial head arthroplasty, the coronoid process was more clearly exposed. Anatomical studies (16) also confirmed that this method could better expose the radial head and coronoid process.

Our team believe that the terrible triad is caused by posterolateral rotational injury; as for other radial head fractures, elbow dislocations combined with anteromedial coronoid process compression fractures caused by varus stress, the articular surface of the coronoid process needs to be reduced and fixed with a buttress plate from the medial side, so they are diagnosed as varus posteromedial instability instead of the terrible triad. The most common coronoid fracture in terrible triad is a tip fracture (17), which is mostly the anterolateral part of the coronoid process, generally not exceeding the sublime tubercle; the ulnar attachments of MCL are often intact, so a medial buttress plate is not usually needed. Due to the coronoid fracture fragments in the terrible triad usually being small and comminuted, the use of screws can easily cause the fragments to break again, increasing the difficulty of the operation and risk of ineffective fixation. The coronoid process can be fixed with 2 Kirschner wires from the dorsal side of the proximal ulna anteriorly and sutured to the anterior joint capsule through 2 bony holes to maintain the anterior stability of the elbow. In cases with small coronoid fragments, a simple fixation with sutures is performed. In patients with severe elbow injuries, the anterior soft tissue is torn from the anterior side of the proximal ulna, resulting in a dislocation tendency of the humeroulnar joint. In these cases, the tension of the anterior joint capsule is more important than the osseous stability of the coronoid process, and this method can achieve satisfactory stability of humeroulnar joint. It is not recommended to resect fragments of radial head in terrible triad, which often leads to postoperative instability. The radial head may be fixed with 2 crossed countersunk screws other than plates after anatomical reduction when possible, so as to reduce implant irritation. If the radial head is severely comminuted or has poor bone density, radial head arthroplasty should be considered. Attention should be given to the height and diameter of the prosthesis in the operation to avoid postoperative instability or "overstuffing" syndrome. Two studies have focused on the clinical results of ORIF versus replacement of the radial head regarding the terrible triad injuries. Watters et al. (18) did not observe any significant differences between groups in terms of ROM and DASH at a minimum of 18 months follow-up. Leigh et al. (19) found that revision surgery was more common in the ORIF group $(5 / 13)$ than in the radial head replacement group (2/11) after a mean follow-up of 41 months.

The repair of the attachments of the LCL complex and the common extensor tendon is critical to postoperative stability (8). They can be repaired by drilling and suturing on the lateral epicondyle or using anchors. It is generally suggested that reconstruction of LUCL should be performed at $40^{\circ}-50^{\circ}$ elbow extension, but when repairing fresh injuries, reconstruction at $90^{\circ}$ elbow flexion and a neutral rotational position of forearm is more convenient (20). Whether to repair MCL is still controversial. Most surgeons believe that it is not necessary to repair MCL, because the extra medial incision will further increase surgical trauma to the soft tissues and cause postoperative complications, especially elbow stiffness $(3,7,15,21)$. The elbow is a triangular stable structure composed of medial, lateral, and anterior parts. After the anterior joint capsule is repaired, flexion-extension stability is established; after the lateral structures are 
reconstructed, rotational stability reappears. The MCLs of patients with the terrible triad often suffer from incomplete injuries, and valgus is generally forbidden during the rehabilitation process, which allows MCLs to heal gradually over 2 months. Therefore, it is usually not necessary to deliberately add a medial incision for repair. After the repair of fractures and soft tissues, the stability of the elbow joint must be verified. An elbow with a full flexion-extension arc under anesthesia without dislocation is considered stable and no stress test is required $(22,23)$. If the elbow is stable intraoperatively, short-term plaster immobilization for within 1 week is sufficient. When the elbow is still unstable after reconstructing the osseous structures and ligaments, a hinged external fixator is applied to protect the repaired bones and soft tissues, maintain the stability of the joint, and enable the patient to mobilize early.

Pugh et al. (13) followed 36 terrible triad patients undergoing internal fixation through the single lateral approach for an average of 34 months, achieving good functional outcomes with a flexion-extension arc of $112^{\circ} \pm 11^{\circ}$ and a rotational range of $136^{\circ} \pm 16^{\circ}$. The average MEPS of these patients was 88 points. Gong et al. (24) compared the outcomes of the single lateral approach with the combined medial and lateral approach for terrible triad of the elbow, and found that the single lateral approach provided better functional results and a lower incidence of postoperative heterotopic ossification.

From 2013 to 2019 , 109 patients with terrible triad were treated through a lateral minimal approach at Beijing Jishuitan Hospital. At a mean time of $36.1 \pm 11.1$ months postoperatively, the flexion-extension ROM of the elbow averaged $123.4^{\circ} \pm 20.7^{\circ}$ degrees and forearm rotation averaged $151.0^{\circ} \pm 25.6^{\circ}$ degrees. The mean MEPS was $92.4 \pm 8.8$ points, with a low reoperation rate of $6.4 \%$, demonstrating satisfactory short-term functional outcomes of the lateral minimal approach.

This study has the following limitations: (I) as a retrospective study, the result is prone to have selection bias; (II) the size of this study, though comparable to or even larger than similar studies, may not be large enough to show the functional outcomes of the lateral minimal approach; (III) the measurement of ROM of elbows was performed by the same doctor, and there may have been favour detection bias.

\section{Conclusions}

Coronoid fractures, radial head fractures, and LCL injuries in the terrible triad of the elbow can be treated satisfactorily through a lateral minimal incision, combined with a hinged external fixation if necessary. The method described above can restore the normal anatomy of the elbow and provide sufficient stability, which may lead to a reduction in the incidence of elbow stiffness by reducing surgical trauma and promoting early mobilization.

\section{Acknowledgments}

Funding: This publication was supported by Beijing Natural Science Foundation (L192049) and Beijing Municipal Health Commission (BMHC2019-9).

\section{Footnote}

Reporting Checklist: The authors have completed the STROBE reporting checklist. Available at https://dx.doi. org/10.21037/atm-21-2542

Data Sharing Statement: Available at https://dx.doi. org/10.21037/atm-21-2542

Conflicts of Interest: All authors have completed the ICMJE uniform disclosure form (available at https://dx.doi. org/10.21037/atm-21-2542). The authors have no conflicts of interest to declare.

Ethical Statement: The authors are accountable for all aspects of the work in ensuring that questions related to the accuracy or integrity of any part of the work are appropriately investigated and resolved. The study was approved by Beijing Jishuitan Hospital Institutional Review Board (No. 201708-04) and written informed consent was obtained from all patients. All procedures performed in this study involving human participants were in accordance with the Declaration of Helsinki (as revised in 2013).

Open Access Statement: This is an Open Access article distributed in accordance with the Creative Commons Attribution-NonCommercial-NoDerivs 4.0 International License (CC BY-NC-ND 4.0), which permits the noncommercial replication and distribution of the article with the strict proviso that no changes or edits are made and the original work is properly cited (including links to both the formal publication through the relevant DOI and the license). See: https://creativecommons.org/licenses/by-nc-nd/4.0/. 


\section{References}

1. Gierer P, Rocher S, Wichelhaus A, et al. Typical fractures and dislocations of the elbow joint and their treatment. Radiologe 2018;58:976-84.

2. Chemama B, Bonnevialle N, Peter O, et al. Terrible triad injury of the elbow: how to improve outcomes? Orthop Traumatol Surg Res 2010;96:147-54.

3. Ring D, Jupiter JB, Zilberfarb J. Posterior dislocation of the elbow with fractures of the radial head and coronoid. J Bone Joint Surg Am 2002;84:547-51.

4. Chan K, MacDermid JC, Faber KJ, et al. Can we treat select terrible triad injuries nonoperatively? Clin Orthop Relat Res 2014;472:2092-9.

5. Chanlalit C, Shukla DR, Fitzsimmons JS, et al. The biomechanical effect of prosthetic design on radiocapitellar stability in a terrible triad model. J Orthop Trauma 2012;26:539-44.

6. Chen HW, Liu GD, Wu LJ. Complications of treating terrible triad injury of the elbow: a systematic review. PLoS One 2014;9:e97476.

7. Rodriguez-Martin J, Pretell-Mazzini J, Andres-Esteban EM, et al. Outcomes after terrible triads of the elbow treated with the current surgical protocols. A review. Int Orthop 2011;35:851-60.

8. Mathew PK, Athwal GS, King GJ. Terrible triad injury of the elbow: current concepts. J Am Acad Orthop Surg 2009;17:137-51.

9. Dodds SD, Fishler T. Terrible triad of the elbow. Orthop Clin North Am 2013;44:47-58.

10. Zhang C, Zhong B, Luo CF. Treatment strategy of terrible triad of the elbow: experience in Shanghai 6th People's Hospital. Injury 2014;45:942-8.

11. Smith MV, Calfee RP, Baumgarten KM, et al. Upper extremity-specific measures of disability and outcomes in orthopaedic surgery. J Bone Joint Surg Am 2012;94:277-85.

12. Cusick MC, Bonnaig NS, Azar FM, et al. Accuracy and reliability of the Mayo Elbow Performance Score. J Hand Surg Am 2014;39:1146-50.

13. Pugh DM, Wild LM, Schemitsch EH, et al. Standard surgical protocol to treat elbow dislocations with radial head and coronoid fractures. J Bone Joint Surg Am 2004;86:1122-30.

14. Lindenhovius AL, Jupiter JB, Ring D. Comparison of acute versus subacute treatment of terrible triad injuries of the elbow. J Hand Surg Am 2008;33:920-6.
15. McKee MD, Pugh DM, Wild LM, et al. Standard surgical protocol to treat elbow dislocations with radial head and coronoid fractures. Surgical technique. J Bone Joint Surg Am 2005;87 Suppl 1:22-32.

16. Desloges W, Louati H, Papp SR, et al. Objective analysis of lateral elbow exposure with the extensor digitorum communis split compared with the Kocher interval. J Bone Joint Surg Am 2014;96:387-93.

17. Doornberg JN, van Duijn J, Ring D. Coronoid fracture height in terrible-triad injuries. J Hand Surg Am 2006;31:794-7.

18. Watters TS, Garrigues GE, Ring D, et al. Fixation versus replacement of radial head in terrible triad: is there a difference in elbow stability and prognosis? Clin Orthop Relat Res 2014;472:2128-35.

19. Leigh WB, Ball CM. Radial head reconstruction versus replacement in the treatment of terrible triad injuries of the elbow. J Shoulder Elbow Surg 2012;21:1336-41.

20. Zeiders GJ, Patel MK. Management of unstable elbows following complex fracture-dislocations--the "terrible triad" injury. J Bone Joint Surg Am 2008;90 Suppl 4:75-84.

21. Forthman C, Henket M, Ring DC. Elbow dislocation with intra-articular fracture: the results of operative treatment without repair of the medial collateral ligament. J Hand Surg Am 2007;32:1200-9.

22. O'Driscoll SW, Jupiter JB, King GJ, et al. The unstable elbow. Instr Course Lect 2001;50:89-102.

23. Gong M, Jiang X. Reflection on the interrelationship of posterior elbow dislocation, posterolateral rotatory injury and the terrible triad of the elbow. J Clin Orthop Res 2020;5:65-7.

24. Gong M, Huang X, Li G, et al. Comparative study of single lateral incision and combined lateral and medial incisions for treatment of terrible triad of the elbow. Chinese Journal of Trauma 2017;33:389-96.

(English Language Editor: J. Jones)

Cite this article as: Zha YJ, Xiao D, Hua KH, Sun WT, Gong MQ, Li T, Chen C, Jiang XY. Lateral minimal approach to the terrible triad of the elbow: a treatment protocol in Beijing Jishuitan Hospital. Ann Transl Med 2021;9(15):1232. doi: 10.21037/atm-21-2542 